\title{
Assessing biosecurity practices, movements and densities of poultry sites across Belgium, resulting in different farm risk-groups for infectious disease introduction and spread
}

\author{
Sarah Van Steenwinkel ${ }^{a}$, Stefaan Ribbens ${ }^{a}$, Els Ducheyne ${ }^{b}$, \\ Els Goossens ${ }^{\mathrm{b}}$, Jeroen Dewulf ${ }^{\mathrm{a}, *}$ \\ a Department of Reproduction, Obstetrics and Herd Health, Veterinary Epidemiology Unit, Faculty of Veterinary Medicine, Ghent University, Salisburylaan \\ 133, B-9820 Merelbeke, Belgium \\ b Avia-Gis, risschotlei 33, B-2980 Zoersel, Belgium
}

\section{A R T I C L E I N F O}

\section{Article history:}

Received 3 September 2010

Received in revised form

26 November 2010

Accepted 7 December 2010

\section{Keywords:}

Hobby poultry

Biosecurity

Between-farm movements

Belgium

Multivariable analysis

Questionnaire

Avian influenza

\begin{abstract}
A B S T R A C T
The existing diversity within poultry systems affects the potential risk of infectious disease introduction and spread. Population data on the level of biosecurity and between-farm contacts is scarce, despite its importance for identifying possible routes of disease transmission. A study was carried out in Belgium to investigate and differentiate professional and hobby poultry sites based on their biosecurity levels and farm movements. Questionnaire data from a total of 37 professional poultry farms, 19 hatcheries and 286 hobby poultry sites were analyzed using a combination of a linear scoring system, a Categorical principal component analysis (CATPCA) and a Two-Step cluster analysis (TSCA). In general, the level of biosecurity was lower in hobby poultry flocks, mainly due to the poor confinement against the outdoor environment and the poor infrastructural hygiene. Most Belgian professional poultry farms and hatcheries had an acceptable level of adoption of standard biosecurity practices, however less attention was given to the way transportation vehicles and employers were brought onto farms and professional visitors welcomed. Considerable variation in the movements and in the structure of the networks arising from these movements was found. Movement frequencies were higher at professional farms compared to hobby farms. Results showed that multiple category farming systems had the highest total movement frequencies. Monthly frequencies of professional visits often exceeded those of poultry and egg movements. Professional and hobby poultry sites were also connected, but movements of poultry and eggs were found only to occur from professional to hobby sites. However, hobby poultry keepers were personally purchasing the poultry and eggs on the professional poultry sites. Six groups of poultry sites were differentiated, which are interpreted as very low to very high risk groups, based on the potential of infectious disease introduction and spread.
\end{abstract}

(c) 2010 Elsevier B.V. All rights reserved.

\section{Introduction}

The probability of disease introduction and spread is determined by a complex combination of factors, such

\footnotetext{
* Corresponding author. Tel.: +32 926475 48; fax: +32 92647557.

E-mail address: Jeroen.Dewulf@UGent.be (J. Dewulf).
}

as the number and density of animals, the type of species or breeds present, the number and type of contacts between flocks, and the sanitary measures that are put in place. To avoid the introduction of diseases into farms and to contain the spread of infections already present, appropriate preventive measures need to be implemented. Such enhancement of biosecurity is generally agreed to be the best way to minimize the risk of 
disease introduction (Boklund et al., 2004; Niemi et al., 2009).

Poultry production is characterized by a huge diversity of production systems, with different scales of production, bird species, measures of biosecurity, production inputs and outputs. Both intensive professional production systems and smallholder hobby poultry sites coexist, with very different characteristics. However, both harbour animals that are susceptible to the same diseases. While the scope and impact of biosecurity measures may be obvious for large-scale poultry production, its significance for small poultry-keeping holdings must not be overlooked; either in their own right or as sources of infection for large professional flocks.

Classification of poultry sites, based on the risk for disease introduction and spread is an important step in the development of risk based surveillance strategies, policies and recommendations for farmers, as well as for modeling purposes (Lyytikäinen and Kallio, 2008; Ortiz-Pelaez and Pfeiffer, 2008; Niemi et al., 2009). For instance, the development of generic risk profiles could help policy makers to direct surveillance and early warning systems towards high-risk holdings and promote measures which reduce the farm's risk of disease introduction. In addition, examining characteristics of poultry sites from the point of view of risk of disease introduction and spread provides useful information for stochastic spatial simulation models, which simulate disease outbreaks.

In spite of the importance of biosecurity and contact structures in disease transmission, there is little information available in the literature on the biosecurity status of poultry farms (Nespeca et al., 1997; East, 2007). There are several papers which have used multivariate analyses to classify livestock farms (Calavas et al., 1998; Solano et al., 2000; Rose and Madec, 2002; Köbrich et al., 2003; Kristensen, 2003; Boklund et al., 2004; Milán et al., 2006; Ribbens et al., 2008; Costard et al., 2009). However, to the author's knowledge no paper has classified different poultry production systems according to their biosecurity practices.

The primary objective of this study was to describe the presently applied biosecurity measures and on/off farm movements of Belgian poultry sites for different flock types, as well as to search for possible links between the professional poultry sector and hobby poultry sites. A secondary objective was to use the output from this biosecurity survey to investigate whether poultry sites could be categorized into risk groups for disease introduction and spread.

The results from this study were used to inform the development of a stochastic spatial model for highly pathogenic avian influenza (HPAI) outbreaks in Belgium (not covered in this article). Therefore, an epidemiological approach towards the investigation of the level of biosecurity required for HPAI was pursued. However, since the majority of poultry pathogens are transmitted via the faeco-oral route, the output from this study can be interpreted as a generic system for risk-classification in relation to disease introduction and spread on poultry farms.

\section{Materials and methods}

\subsection{Selection of poultry premises}

The target population for this study comprised both professional and hobby poultry sites in Belgium.

A list of all Belgian professional poultry operations was available from an identification and registration database of animals (SANITEL-Poultry). This database provides information on the type of operation, the maximum capacity for each bird species kept and the geographical coordinates of the farm location. Professional poultry holdings in this database are defined as premises where more than 200 birds are kept at the same location. This relates to the regulatory obligation to report each poultry premise with more than 200 birds. Eighty professional poultry operations were randomly selected from the SANITEL database, corresponding to a sampling fraction of $4.4 \%$. A stratified proportional allocation was performed to assure a good representation of the major animal species, types of operations and a representative geographical distribution. Strata were created based on: (1) animal species (chicken, duck, pigeon, pheasant, turkey, quail, guinea fowl and partridge), (2) type of operation (rearing, multiplier hens, layer hens, broilers, selection and show) and (3) geographical location. In addition, all 32 existing recognized hatcheries were selected.

For smallholder poultry sites (<200 birds) no official database exists. However, in early 2006 all municipalities (smallest administrative units in Belgium) in Belgium were asked - as a precautionary measure due to the potential HPAI threat - to identify all inhabitants that kept hobby birds. Two hundred and eight municipalities which surround the selected professional poultry operations and hatcheries were contacted and asked whether they still had this hobby poultry inventory and were willing to give access to the database. A municipality was considered to surround a particular professional operation when its area overlapped with a $3 \mathrm{~km}$ radius around the farm. Seventy municipalities (34\%) responded positively, of these 55 provided a full dataset corresponding to 27,766 hobby poultry keepers. All addresses were geocoded and 2000 hobby poultry holdings, lying within a $3 \mathrm{~km}$ radius of a professional poultry operation were randomly selected to receive a mailed questionnaire (see further).

\subsection{Description and location of professional and hobby poultry premises}

Type of farm was represented by seven categories: broiler, layer, breeder, multiplier, multiple-category farms, hatcheries and hobby farms. The capacity of the farm was divided into three possible sizes: small, medium and large, using as cut-off points, the 33.3 and 66.6 percentile of the maximum capacity of all professional poultry operations from the SANITEL database. All hobby poultry sites were considered small. The professional poultry farm density of the region was measured using three categories: sparsely populated poultry farm area (SPPA), medium populated poultry farm area (MDPA) and densely populated poultry farm area (DPPA). To this end, the point locations were con- 
verted into a continuous raster using the quadratic kernel density estimation function (Spatial Analyst, ArcMap 9.3, ESRI, Redlands, CA, USA). Bandwidth selection for the kernel smoothing was $7.5 \mathrm{~km}$. Output cell size was $900 \mathrm{~m}$. The resulting density plots expressed the number of professional poultry farms per $\mathrm{km}^{2}$. We defined the 3 density classes based on the Jenk's natural breaks classification method, which is a data classification method designed to determine the best arrangement of values into different classes. This is done by seeking to minimize each class's average deviation from the class mean, while maximizing each class's deviation from the means of the other groups (ArcMap 9.3, ESRI FAQ).

Based on the database of 27,766 geocoded hobby poultry premises the mean distance to the nearest neighbour was calculated using ArcGIS (ArcMap 9.3, ESRI, Redlands, CA, USA).

\subsection{Administration of the questionnaire}

The 80 selected professional poultry farms were subjected to a questionnaire interview by telephone. Additionally, a questionnaire was administered to the 32 hatcheries by regular mail. The 2000 hobby poultry holdings were contacted by regular mail and asked to complete an online questionnaire. The surveys were conducted between September and December 2008. All telephone interviews, including the coding and typing of the information, were conducted by the first author.

To increase the response rate, an incentive was given: each participant with a hobby poultry holdings received a voucher worth $€ 2$ redeemable with purchase of poultry feed. In addition, 50 gift vouchers worth $€ 10$ were included in a raffle. All professional poultry farms that participated were offered a free yearly subscription to a professional poultry magazine.

\subsection{Questionnaire design}

For each type of poultry premises an adapted questionnaire was designed, both in Dutch and French. A preliminary draft was pre-tested on 8 hobby poultry farmers, 1 professional poultry farm and 3 experts. The questionnaire was divided into four parts and included both standardised closed and semi-closed questions, in total consisting of 9 pages:

1. General data: identification, type of farm, capacity, bird species, presence of other animals.

2. Infrastructure: presence of farm fences, boot dips, sanitary transition zone, paved (un-) loading place, freerange and housing secure against wild birds.

3. Hygiene: cleaning and disinfection, all-in-all-out, pest control, access of wild birds to fresh litter and manure, access of rodents and wild birds to feed storage, feeding outside, type of drinking and cleaning water, allocation of waste water, dead bird disposal, employees and visitors.

4. Contacts/movements: this part provided a table of all types of external contacts that could be applicable to the poultry premise (poultry supply and discharge, sup- pliers of food and litter, veterinarians, pest control, disinfection, manure and dead bird removal, advisors and controlees, hobby poultry keepers, local vendors, employees). Each participant was asked to select those external contacts that were applicable to their situation and to complete the table with the frequency for each type of contact, how many persons were involved, whether they accessed the bird compartments and whether they wore company clothing. Contacts with non-professionals such as neighbors, friends, family were not taken into account as long as they did not own poultry themselves as it was believed that these were dead end contacts and therefore do not contribute to potential disease spread.

The questionnaire (in Dutch or French) can be obtained upon request.

\subsection{Data processing}

All information was coded numerically to assist analysis, entered into a database worksheet program (Microsoft Excel, 2007) and recoded into categorical data (nominal and ordinal level) for further analysis.

\subsection{Data analysis}

\subsubsection{Creating a biosecurity scoring system}

Variables which were assumed to have a similar influence on the potential risk of introduction of contagious disease on the farm were combined into a single variable, thereby producing a basic biosecurity score. To this end, all variables were coded using values of 1 (biosecurity measure present) or 0 (absent). All variables were divided into groups, each expressing a different aspect of farm biosecurity. Then, for each biosecurity variable group (made up of several measures) the values for each individual variable were added up to generate a biosecurity score. Finally, each group score was scaled from 0 to 10 , except for one group ('susceptibility of birds', see below). Thus, a higher score implies a 'better' biosecurity level for the variable group concerned.

The following biosecurity variable groups were considered:

1. Susceptibility of birds: based on their susceptibility to HPAI, two groups of bird species were defined (Alexander, 2007; Sharkey et al., 2008): highly susceptible and low susceptibility species. Chickens, turkeys, pheasants, partridges and mixed bird species were allocated to the highly susceptible group. Ducks, geese, pigeons and ostriches were in the low susceptibility group. The hatching eggs of hatcheries were also in the low susceptibility group (Ligon, 2005; Alexander, 2007).

2. Other animals: this group represented variables referring to the presence of other animals such as pets, production animals, hobby poultry and rodents.

3. Wild birds: this included all measures that are aimed at preventing (in)direct contact with wild birds, such as secure housing, wild birds having no access to fresh 
litter/manure/food storage and no surface water being used.

4. Cleanliness of infrastructure: this referred to permanent structures within the farm or activities which were part of the daily management (presence of boot dips, sanitary transition zone, cleaning and disinfection, dead bird disposal, etc.).

5. Hygiene of persons: this included measures for reducing the number and intensity of direct contacts between poultry and external persons (no visitors allowed in poultry houses/free-range, company clothing provided to all kinds of (professional) visitors).

6. Hygiene of transport: this related to information on biosecure transportation of poultry or poultry products (trucks without contact with other poultry farms in one day, single purpose of transportation truck, company transport material when selling to local traders or hobby poultry keepers).

The information on the external contact types and frequencies was separated into 'on-farm' and 'off-farm' movements, followed by a subdivision into 'living poultry', 'poultry products' (eggs and manure) and 'fomites' (inanimate objects which are capable of transmitting infectious organisms). The monthly frequencies were coded into 'no', 'low', 'medium' and 'high' movement frequencies. In this way, a score was defined on a scale from 1 to 4 , with a higher score implying lower movement frequencies (more secure against infectious agents).

\subsubsection{Categorical principal components analysis (CATPCA)}

To analyze the categorical data, the CATPCA procedure was used (SPSS 15.0). The optimal scaling process transformed the categorical variables into metric variables, by means of monotonic optimal least squares transformations. The results of a CATPCA can be presented using a graphical display. The component loadings are correlations between the variables and the components (dimensions), and they are used as coordinates to represent the variables as vectors in the component space on a 2-dimensional display. The squared distance of the vector tip to the origin corresponds to the percentage of variance accounted for (PVAF). If the PVAF for two variables is adequate, a small angle between the two vectors in the space indicates a large correlation between the two variables. The theory of CATPCA is described, among others, in Meuleman et al. (2004) and Linting et al. (2007).

All scored variables were given an ordinal measurement scale in the analysis. Three supplementary variables were included and attributed a multiple nominal measurement scale (i.e., as grouping variables) (Linting et al., 2007): type of farm, capacity of the farm and professional poultry-farm density of the region. The quantification of a supplementary variable has no influence on the actual analysis, but allows the interpretation of its relationship with the result obtained for the other variables.

In our analyses, the number of dimensions was set at a default value of 2 , which consequently allowed for two-dimensional graphical representation. The reduction to two dimensions was acceptable since the sum of PVAF, which is a measure of model fit, was largely sufficient. Because $44 \%$ of the respondents included one or more missing values on these 12 scored variables, a missing data treatment strategy other than deleting all cases with at least one missing data value was required. It was decided to use the straightforward CATPCA option of imputing the modal category for each of the variables (Ferrari and Anonni, 2005). Finally, the variable principal normalization option was used, which optimizes the association between variables.

\subsubsection{Two-step cluster analysis (TSCA)}

The object scores obtained from the CATPCA solutions were then included in a two-step cluster analysis (TSCA, SPSS 15.0) to identify clusters of poultry systems with a similar biosecurity level and on-to/off farm movement frequencies (Ribbens et al., 2008).

\section{Results}

General hobby poultry flock characteristics are shown in Table 1 . The most frequently kept poultry species were chickens (85\%), pigeons (12\%), geese (10\%) and ducks (9\%). Eighty-six percent of the hobby flocks with chickens had fewer than 10 birds. Pigeon flocks were generally larger, with a median flock size of 25 birds. The average distance between the hobby poultry farms in the 55 municipalities was $110.7 \mathrm{~m}$ (median $=81.1 \mathrm{~m}$ ). The density varied between 1.7 and 39.1 premises per $\mathrm{km}^{2}$ with an average of 12.4 . The proportion of hobby poultry flocks per inhabitant in these 55 municipalities varied between 0.5 and $10.2 \%$ with an average of 4.0. Given that the average number of persons per household in Belgium is estimated to be 2.3 this relates to an average of $9.2 \%$ of the households having hobby poultry.

\subsection{Survey response}

Of the 80 selected professional poultry farms, 78 registered addresses and telephone numbers were valid; responses were received from 48 (61.5\%) of them, but only 37 (47.4\%) questionnaire forms were completed because 11 farms had stopped production. These 37 farms represented 13 of the 24 different species * production type combinations present in the study population. For the hatcheries a response rate of $59.4 \%$ was obtained (19/32). The questionnaires for hobby poultry holdings were mailed to 2000 addresses, of which 1905 (95.3\%) were valid and of these $373(19.6 \%)$ responded. Eighty seven respondents no longer raised poultry, resulting in 286 (15.0\%) complete questionnaires. All participants are geographically represented in Fig. 1.

Of the total population of professional poultry farms, $56 \%$ consist of broilers, $19 \%$ of layers, $8 \%$ of multipliers, $10 \%$ of rearing farms, $1 \%$ of show and $5 \%$ of poultry farms with multiple category activities. Similar proportions were obtained among the respondents: $51 \%$ broilers, $11 \%$ layers, $11 \%$ multipliers, $8 \%$ rearing farms. Only for the group of farms with multiple production type activities, a higher 
Table 1

Flock characteristics of the selected hobby poultry premises.

\begin{tabular}{|c|c|c|c|c|c|c|c|c|}
\hline & \multirow{2}{*}{$\begin{array}{l}\% \text { of flocks with } \\
\text { specified species }\end{array}$} & \multirow{2}{*}{$\begin{array}{l}\text { Flock size median } \\
\text { [min.-max.] }\end{array}$} & \multicolumn{6}{|c|}{ Percentage keeping this number of birds } \\
\hline & & & {$[1-10]$} & {$[11-20]$} & {$[21-30]$} & {$[31-50]$} & {$[51-100]$} & $>100$ \\
\hline Chicken & 85.8 & $5[1-100]$ & 86.2 & 10.8 & 1.9 & 0.8 & 0.2 & 0.0 \\
\hline Pigeon & 12.1 & $25[1-200]$ & 34.3 & 12.8 & 7.2 & 6.4 & 23.1 & 9.2 \\
\hline Goose & 10.3 & $3[1-80]$ & 95.5 & 3.5 & 0.7 & 0.2 & 0.1 & 0.0 \\
\hline Duck & 9.2 & $3[1-100]$ & 90.2 & 7.1 & 1.6 & 0.5 & 0.3 & 0.0 \\
\hline Other $^{\mathrm{a}}$ & 8.7 & $6[1-260]$ & 63.3 & 18.4 & 7.0 & 3.4 & 4.3 & 1.3 \\
\hline Pheasant & 2.4 & $3[1-110]$ & 90.6 & 7.0 & 1.3 & 0.6 & 0.3 & 0.1 \\
\hline Peacock & 2.0 & $2[1-100]$ & 95.6 & 3.2 & 0.7 & 0.4 & 0.2 & 0.0 \\
\hline Quail & 1.5 & $3[1-182]$ & 90.5 & 4.7 & 1.9 & 0.5 & 0.5 & 0.2 \\
\hline Guinea fowl & 1.1 & $3[1-200]$ & 70.5 & 4.2 & 3.6 & 4.5 & 10.4 & 1.3 \\
\hline Turkey & 0.9 & $2[1-48]$ & 97.1 & 1.2 & 1.2 & 0.0 & 0.0 & 0.0 \\
\hline Running bird & 0.5 & $2[1-54]$ & 94.6 & 3.4 & 0.7 & 0.0 & 0.7 & 0.0 \\
\hline Partridge & 0.2 & $3[1-62]$ & 86.5 & 7.7 & 0.0 & 0.0 & 1.9 & 0.0 \\
\hline Swan & 0.2 & $2[1-48]$ & 95.3 & 3.1 & 0.0 & 0.0 & 0.0 & 0.0 \\
\hline Total & & $6[1-500]$ & 72.7 & 13.4 & 4.3 & 2.4 & 4.0 & 1.7 \\
\hline
\end{tabular}

a Such as birds of prey and other aviary birds kept outside the house.

proportion was received (19\%). Most poultry farms are situated in the northern part of the country (79\%), with the highest proportion in the provinces of West-Flanders (30\%) and Antwerp (21\%). The responding farms showed approximately the same geographical distribution throughout the country. General characteristics of the studied population sample, regarding the bird species kept or hatched, size of the farm and professional poultry population density of the area, are summarized in Table 2.

\subsection{Biosecurity}

All professional poultry farms in the sample had birds present at the farm, and therefore were susceptible to HPAI. In contrast, hatcheries were not considered susceptible, as hatching eggs are not expected to be able to produce infected one day old chickens. The possibility of indirect infection of the one day old chicks through environmental contamination in the hatcheries was ignored. Ninety-four

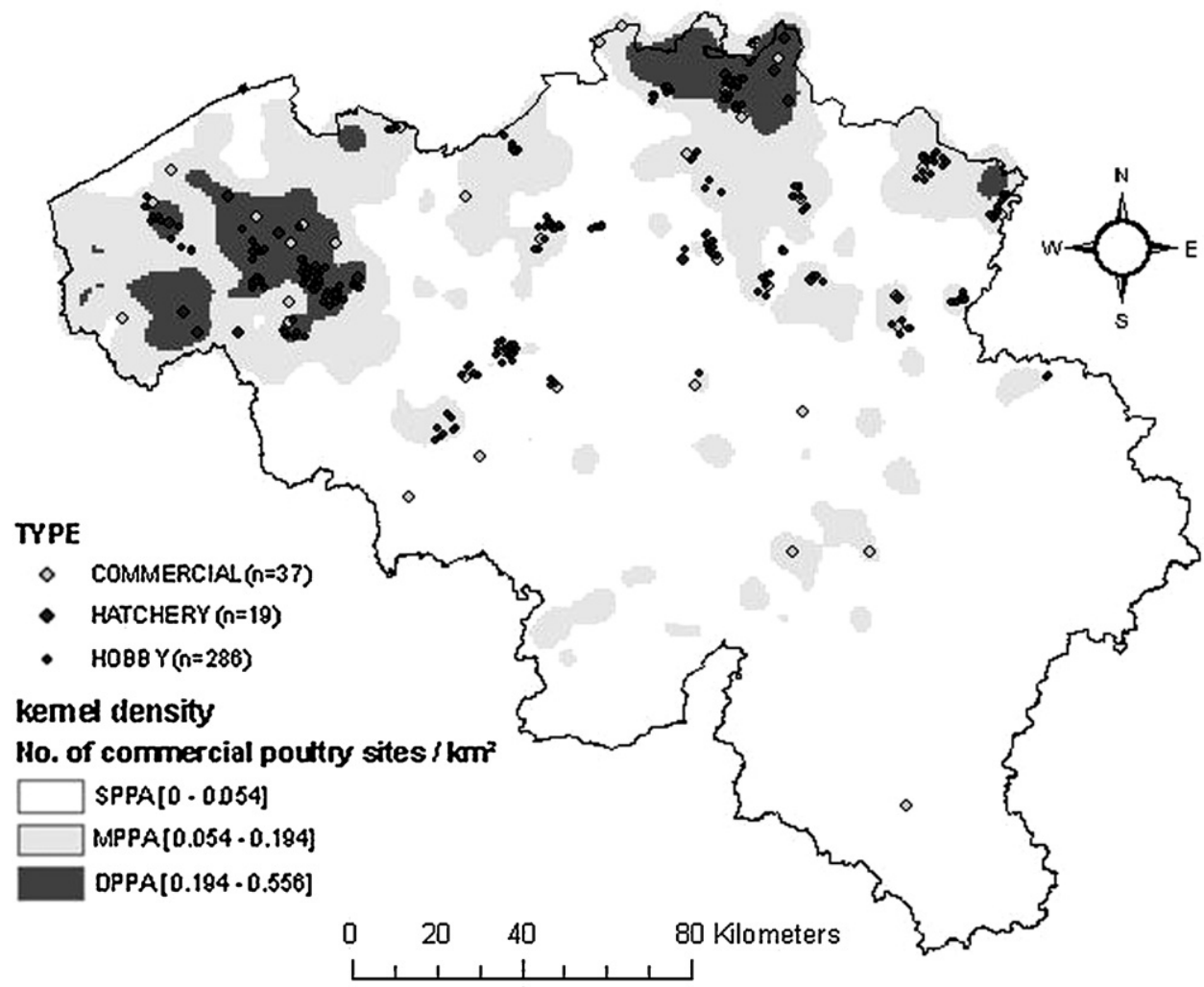

Fig. 1. Geographical presentation of participants in the 2008 biosecurity survey, Belgium. In the background, the spatial kernel density estimates (bandwidth $7.5 \mathrm{~km}$; raster size $0.9 \mathrm{~km}$ ) of all professional poultry sites are given. 
Table 2

General characteristics of the studied population sample, Belgium 2008.

\begin{tabular}{|c|c|c|c|}
\hline & Professional farms $(n=37)(\%)$ & Hatcheries $(n=19)(\%)$ & Hobby poultry premises $(n=286)(\%)$ \\
\hline \multicolumn{4}{|l|}{ Bird species } \\
\hline Pigeon & & & 5 \\
\hline Duck & & & 1 \\
\hline Pheasant & 3 & 11 & \\
\hline Goose & & & 1 \\
\hline Turkey & 3 & & \\
\hline Chicken & 68 & 68 & 62 \\
\hline Ostrich & & 11 & \\
\hline Partridge & & 5 & \\
\hline Multiple species & 27 & 5 & 31 \\
\hline \multicolumn{4}{|l|}{ Size $^{\mathrm{a}}$} \\
\hline Small & 38 & 32 & 100 \\
\hline Medium & 43 & 26 & \\
\hline Large & 19 & 42 & \\
\hline \multicolumn{4}{|l|}{ Density ${ }^{\mathrm{b}}$} \\
\hline SPPA $^{\mathrm{c}}$ & 38 & 5 & 26 \\
\hline MPPA $^{\mathrm{d}}$ & 41 & 21 & 39 \\
\hline DPPA $^{\mathrm{e}}$ & 22 & 74 & 35 \\
\hline
\end{tabular}

a 33.3 and 66.6 percentile of the maximum capacity of all professional poultry operations from the SANITEL database.

b Based on a spatial kernel density estimate of the number of professional poultry sites per km² (bandwidth $7.5 \mathrm{~km}$; raster size $0.9 \mathrm{~km}$, density classes based on natural breaks, 'Jenk's').

c Sparsely populated poultry farm area.

d Medium populated poultry farm area.

e Densely populated poultry farm area.

percent of the hobby poultry premises kept highly susceptible bird species for HPAI. Table 3 shows the percentage of farms implementing different types of biosecurity measures, for each of the three types of poultry operations, together with the mean biosecurity score for each group.

Several contacts between professional farms and both poultry traders and hobby poultry keepers were identified: $46 \%$ trade live poultry (and their products) with local traders and $38 \%$ trade live poultry (and their products) with hobby poultry keepers. Also $16 \%$ of the hatcheries traded with local traders and even 58\% traded with hobby poultry keepers (hatching eggs or one day old chickens). Eleven and nineteen percent of the respondents at hatcheries and professional poultry farms, respectively, stated they visited bird shows on average once a year, but without selling or purchasing birds. Twenty-one percent of the hobby poultry keepers visited bird shows more often (on average 6.3 times/year) and $8 \%$ also sold or purchased birds at these bird shows.

Hobby poultry keepers ranked egg production and processing kitchen waste as the most common reason for keeping birds. The majority of the hobby poultry keepers (77\%) would implement preventive measures (creating a covered and fenced outdoor scavenging area or to put all poultry indoors) if required to do so by the government.

\subsection{Movements}

Fig. 2 shows the contact structure between professional poultry farms, hatcheries, traders and hobby poultry keepers, arising from the trade of live poultry or (hatching) eggs. For each contact, the movement direction and the average monthly frequency are shown. In addition, extra movements occurred resulting from farm visits by veterinarians, feed and litter suppliers, vaccinators, rodent control teams, manure removal trucks, cleaning and disinfection teams, dead bird/rotten eggs disposal trucks, control agencies, repairers and advisors. A summary of these is provided in Table 4. Multiple production type/purpose farms had on average the highest total movement frequencies (46.4/month), mainly resulting from the high frequency of off farm trade of live poultry. Hatcheries had a mean of 25.3 onto and off farm movements per month, and showed the largest frequencies of monthly professional visitors (14.4/month). The majority of hobby poultry sites rarely had off-farm movements, however exceptions occurred.

The survey also examined the distances travelled by hobby poultry keepers to purchase live poultry or (hatching) eggs from professional poultry farms and hatcheries. The percentage of hobby poultry keepers travelling certain distances were as follows: $6.4 \%$ [ $<1 \mathrm{~km}], 40.4 \%$ [2-5 km], $10.6 \%$ [6-10 km], 27.7\% [11-20 km], 8.5\% [21-30 km], 2.1\% [31-50] and $4.3 \%$ [>50 km].

Categorical principal components analysis and two-step cluster analysis: The result of a two-dimensional solution of the CATPCA explained 56.6\% of the variance of the scores provided by the 342 respondents for the 12 variables. The percentage of variance accounted for (PVAF) in the first dimension (40.3\%) was more than two times the PVAF in the second dimension (16.3\%). Fig. 3 shows the plot of component loadings, together with the centroid coordinates of the multiple nominal category points. The vectors (lines) are relatively long (always between -1 and 1 ), indicating that the first two dimensions accounted for most of the variance of all of the quantified variables.

The vector of a variable, points into the direction of the highest category of the variable, in this case indicating a higher level of biosecurity or lower on/off farm movement frequencies. The active variables in Fig. 3 approximately form 4 groups, each appearing in a different quadrant. 
Table 3

Mean biosecurity-score per category (scoring index on 10 points; the higher the score, the better biosecurity level) and per type of production system.

\begin{tabular}{|c|c|c|c|c|}
\hline Cat. & Biosecurity components & $\operatorname{COMM}(n=37)$ & $\operatorname{HAT}(n=19)$ & $\operatorname{HOB}(n=286)$ \\
\hline \multirow[t]{7}{*}{ Other animals } & No other farm animals present & 3.2 & 8.4 & 7.8 \\
\hline & No pets present & 4.6 & 6.3 & 4.0 \\
\hline & No hobby poultry present (hatchery = live poultry) & 9.5 & 3.2 & $p^{\mathrm{a}}$ \\
\hline & No contacts between poultry and other animals & 9.1 & 10.0 & 4.7 \\
\hline & Permanent rodent control & 8.4 & 9.5 & 7.2 \\
\hline & Stored feed is not accessible to rodents & 8.9 & 1 & 9.0 \\
\hline & Score (mean; SD) & $(7.3 ; 1.6)$ & $(7.9 ; 1.4)$ & $(7.1 ; 1.6)$ \\
\hline \multirow[t]{10}{*}{ Wild birds } & Poultry areas not accessible to wild birds & 7.6 & 10.0 & 1.9 \\
\hline & Used cleaning water is not drained outside (open) & 7.0 & 8.3 & 1 \\
\hline & Wild birds have no access to stored fresh litter & 8.4 & 1 & 7.6 \\
\hline & Permanent wild bird control (chasing, shooting) & 0.0 & 1.6 & 6.0 \\
\hline & Wild birds have no access to stored manure & 5.7 & 1 & 4.9 \\
\hline & Wild birds have no access to stored food & 9.7 & 1 & 9.2 \\
\hline & No feeding outside and no access to it by wild birds & 9.2 & 1 & 3.2 \\
\hline & Surface water is not used for drinking & 10.0 & I & 8.3 \\
\hline & Surface water is not used for cleaning & 10.0 & 8.9 & 10.0 \\
\hline & Score (mean; SD) & $(7.9 ; 1.3)$ & $(8.9 ; 0.8)$ & $(6.3 ; 1.7)$ \\
\hline \multirow{11}{*}{$\begin{array}{l}\text { Hygiene } \\
\text { infrastructure }\end{array}$} & Fence present around the farm yard perimeter & 6.8 & 6.8 & 0.0 \\
\hline & Boot dips present & 8.6 & 8.4 & 0.0 \\
\hline & Sanitary transition zone(s) present & 9.2 & 7.4 & 0.0 \\
\hline & Presence of paved place of (dis)charge & 9.7 & 10.0 & 0.0 \\
\hline & No multiple ages are kept together & 8.4 & 5.8 & 0.0 \\
\hline & No partial depopulation & 6.2 & 1 & 1 \\
\hline & Regular cleaning and disinfection & 9.5 & 10.0 & 5.0 \\
\hline & Proper cleaning and disinfection of egg containers & 8.9 & 8.9 & 1 \\
\hline & Proper disposal of dead birds & 5.7 & 6.8 & 2.7 \\
\hline & Staff no contact with other poultry (farms) & 9.2 & 6.3 & 1 \\
\hline & Score (mean; SD) & $(8.3 ; 1.1)$ & $(8.0 ; 1.6)$ & $(3.7 ; 0.7)$ \\
\hline \multirow{6}{*}{$\begin{array}{l}\text { Hygiene } \\
\text { persons }\end{array}$} & Visitors no access to poultry compartments & 8.1 & 6.3 & 0.0 \\
\hline & Good hygiene $^{\mathrm{b}}$ of supply teams & 6.5 & 9.5 & 1 \\
\hline & Good hygiene of discharge teams & 6.5 & 10.0 & 1 \\
\hline & Good hygiene of professionals & 7.6 & 6.3 & 1 \\
\hline & Good hygiene of control agencies & 6.2 & 5.8 & 1 \\
\hline & Score (mean; SD) & $(8.6 ; 0.1)$ & $(8.8 ; 1.0)$ & $(0.0 ; 0.0)$ \\
\hline \multirow{6}{*}{$\begin{array}{l}\text { Hygiene } \\
\text { transport }\end{array}$} & Transp. vehicles do not visit more than 1 farm/day & 5.6 & 4.7 & 1 \\
\hline & Transp. vehicles are not used for double purpose & 9.0 & 5.0 & 1 \\
\hline & Poultry (products) not sold to several companies & 7.1 & 0.0 & 1 \\
\hline & Hygienic trade ${ }^{c}$ of poultry/products to traders & 8.1 & 10.0 & $9.2^{\mathrm{d}}$ \\
\hline & Hygienic trade of poultry/products to persons & 7.8 & 10.0 & 10.0 \\
\hline & Score (mean; SD) & $(8.7 ; 1.3)$ & $(7.2 ; 1.3)$ & $(9.9 ; 0.3)$ \\
\hline
\end{tabular}

COMM: professional poultry farms; HAT: hatcheries; HOB: hobby poultry premises.

a Not applicable.

b Wearing company clothing when entering poultry.

c Trader/private person does not use own transport material.

d No selling/buying at bird shows.

Table 4

Mean number of monthly contacts on a poultry site, attributable to on- and off-farm movements of live poultry (hatching) eggs and professional visitors, 2008.

\begin{tabular}{|c|c|c|c|c|c|}
\hline & On poultry/ hatching eggs & Off poultry & Off eggs & Professionals & Total (on and off) \\
\hline \multicolumn{6}{|c|}{ Mean freq./mo. [min., median, max.] } \\
\hline Multiplier & $\begin{array}{l}0.08 \\
{[0.08 ; 0.08 ; 0.08]}\end{array}$ & $\begin{array}{l}0.48 \\
{[0.08 ; 0.08 ; 1.67]}\end{array}$ & $\begin{array}{l}5.58 \\
{[0.08 ; 7.08 ; 8.08]}\end{array}$ & $\begin{array}{l}6.21 \\
{[1.67 ; 7.54 ; 8.08]}\end{array}$ & $\begin{array}{l}12.35 \\
{[3.49 ; 14.79 ; 16.33]}\end{array}$ \\
\hline Hatchery & $\begin{array}{l}1.76 \\
{[0.08 ; 1.76 ; 4.33]}\end{array}$ & $\begin{array}{l}5.98 \\
{[0.42 ; 5.98 ; 20.83]}\end{array}$ & $\begin{array}{l}3.2 \\
{[0.17 ; 3.20 ; 13.33]}\end{array}$ & $\begin{array}{l}14.38 \\
{[0.13 ; 10.96 ; 50.71]}\end{array}$ & $\begin{array}{l}25.32 \\
{[7.44 ; 22.58 ; 54.54]}\end{array}$ \\
\hline Rearing & $\begin{array}{l}0.25 \\
{[0.21 ; 0.21 ; 0.33]}\end{array}$ & $\begin{array}{l}0.47 \\
{[0.21 ; 0.21 ; 1.00]}\end{array}$ & & $\begin{array}{l}5.31 \\
{[1.33 ; 6.29 ; 8.29]}\end{array}$ & $\begin{array}{l}6.03 \\
{[2.67 ; 6.71 ; 8.71]}\end{array}$ \\
\hline Broiler & $\begin{array}{l}0.50 \\
{[0.25 ; 0.54 ; 0.58]}\end{array}$ & $\begin{array}{l}1.02 \\
{[0.25 ; 0.58 ; 2.75]}\end{array}$ & & $\begin{array}{l}7.54 \\
{[4.00 ; 6.18 ; 13.92]}\end{array}$ & $\begin{array}{l}9.06 \\
{[5.50 ; 8.85 ; 15.42]}\end{array}$ \\
\hline Layer & $\begin{array}{l}0.17 \\
{[0.08 ; 0.13 ; 0.33]}\end{array}$ & $\begin{array}{l}0.78 \\
{[0.08 ; 0.43 ; 2.17]}\end{array}$ & $\begin{array}{l}6.06 \\
{[4.00 ; 6.08 ; 8.08]}\end{array}$ & $\begin{array}{l}10.78 \\
{[4.54 ; 9.42 ; 19.75]}\end{array}$ & $\begin{array}{l}17.79 \\
{[10.03 ; 15.52 ; 30.08]}\end{array}$ \\
\hline Multiple category & $\begin{array}{l}1.75 \\
{[0.17 ; 1.75 ; 3.33]}\end{array}$ & $\begin{array}{l}18.99 \\
{[1.67 ; 12.50 ; 68.75]}\end{array}$ & $\begin{array}{l}20.87 \\
{[0.08 ; 20.87 ; 41.67]}\end{array}$ & $\begin{array}{l}4.81 \\
{[1.41 ; 3.96 ; 9.08]}\end{array}$ & $\begin{array}{l}46.42 \\
{[20.83 ; 37.29 ; 121.33]}\end{array}$ \\
\hline Hobby poultry & $\begin{array}{l}0.10 \\
{[0.01 ; 0.03 ; 4.33]}\end{array}$ & $\begin{array}{l}0.09 \\
{[0.00 ; 0.00 ; 4.33]}\end{array}$ & & $\begin{array}{l}1.58 \\
{[0.00 ; 0.08 ; 45.51]}\end{array}$ & $\begin{array}{l}1.77 \\
{[0.01 ; 0.15 ; 45.56]}\end{array}$ \\
\hline
\end{tabular}




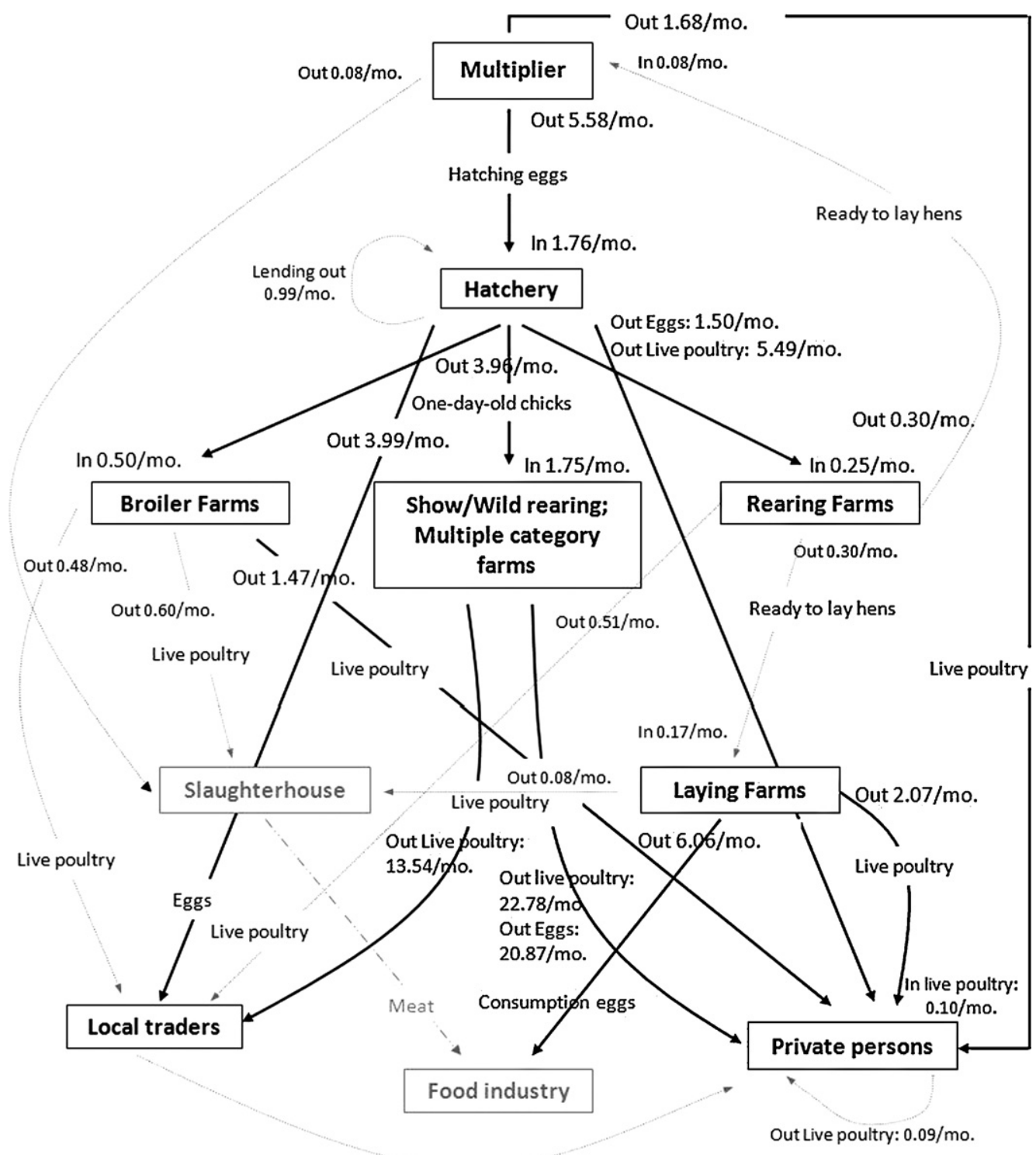

Fig. 2. Contact structure between professional poultry farms, hatcheries, local traders and hobby poultry keepers, arising from the trade of live poultry or (hatching) eggs. For each contact, the movement direction and the average monthly frequency is shown.

Based on survey, 2008

The orientation of the vectors for the variables in the first and the third groups is approximately the same, but the vectors point in opposite directions, indicating a strong negative relationship between these groups of variables. The same conclusions can be made for the variables in the second and the fourth group. Examining category points for the variable size, we see that the first dimension reveals the contrast between small, medium and large poultry farms, whereby the latter show high frequencies of onto and off farm movements (lower score) relative to small farms. However, large farms have a high level of infrastructure hygiene (higher score) relative to small farms. Medium-size poultry farms generally also exert a high level of infrastructural hygiene, as large farms do. However, they show low frequencies of on farm movements of animal products and a more hygienic way of transportation. So, dimension 2 distinguishes between medium-size and large farms.

The object scores obtained from the CATPCA solution, together with the solutions of the two consecutive twostep cluster analysis (TSCA), are presented in Fig. 4. A first TSCA revealed 3 clusters in the sample population. Because there was a larger variation within the sample of the professional poultry farms and hatcheries than in the sample of the hobby poultry premises, a single TSCA was unable to reveal subgroups in an aggregated analysis. Therefore, the object scores of the 3 different clusters were subjected to a second TSCA. This additional analysis revealed 2 sub-clusters within the former cluster. 


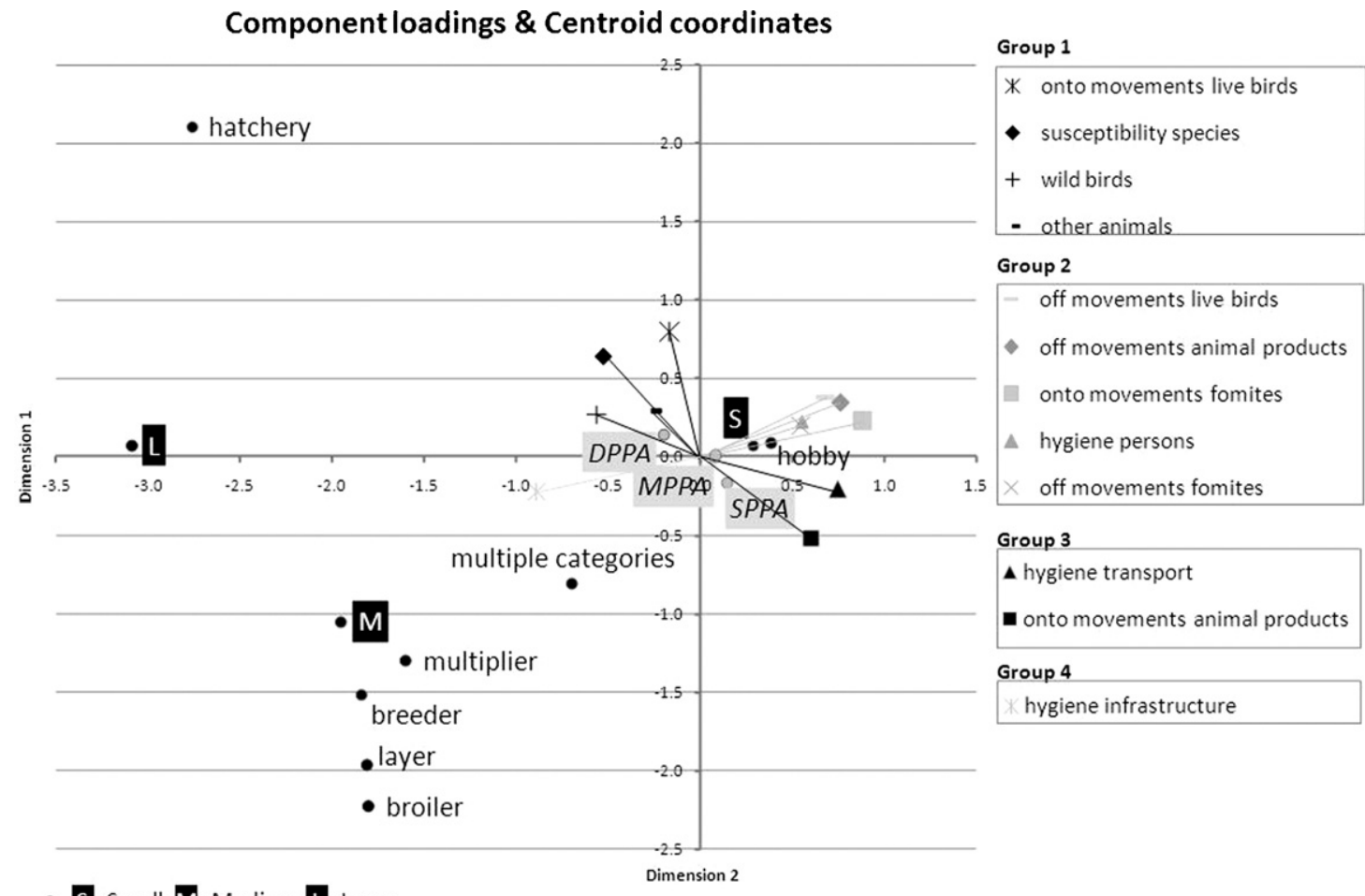

- S: Small; M: Medium; L: Large

- SPPA/MPPA/DPPA:Sparsely/Medium/Densely populated poultry area

Fig. 3. Biplot of component loadings for the active variables and multiple nominal category points, CATPCA analysis. Based on survey, 2008.

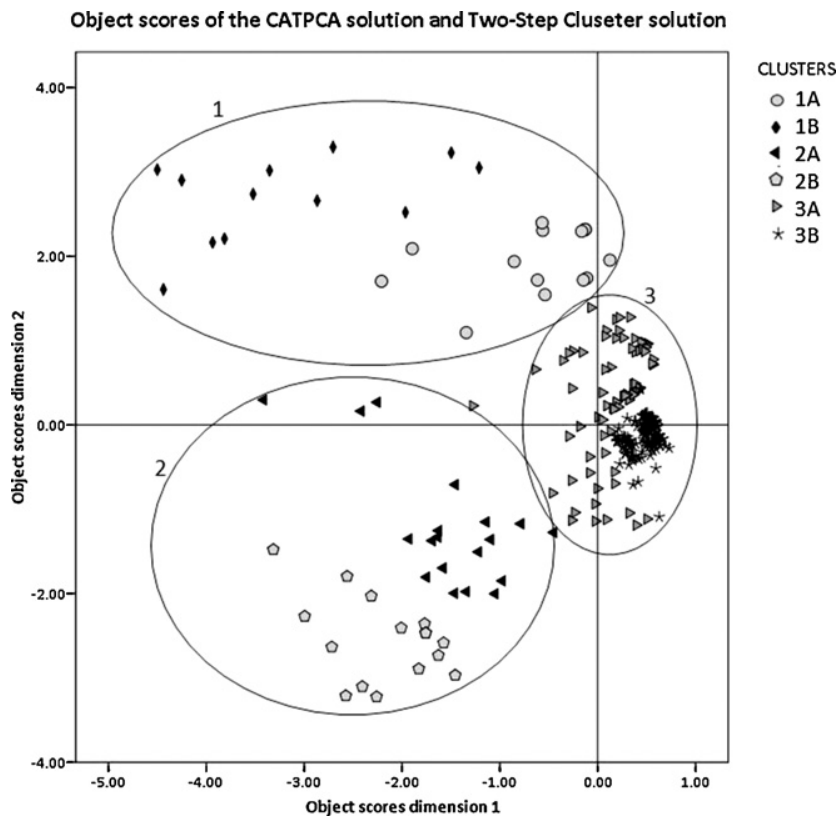

Fig. 4. Object scores of the CATPCA solution and two-step cluster solution. Cluster 1 is the low risk group with $1 \mathrm{~A}=$ Very low risk group and $1 \mathrm{~B}=$ low risk group. Cluster 2 is the high risk group with $2 \mathrm{~A}=$ high risk group and $2 \mathrm{~B}=$ very high risk group. Cluster 3 is the medium risk group with $3 \mathrm{~A}=$ slightly reduced risk group and $3 \mathrm{~B}=$ slightly increased risk group. 
1. Very low risk group - Cluster $1 \mathrm{~A}(n=14)$ : This cluster consisted of hobby poultry premises (71\%) and small to medium size hatcheries (29\%). These sites were characterized by absence of birds susceptible to HPAI virus, a high standard of confinement facilities for birds (isolating against exposure to other animals, birds and visitors), but a rather low level of infrastructural hygiene standard. They were also characterized by very few on- and off- farm movements.

2. Low risk group - Cluster $1 \mathrm{~B}(n=12)$ : All sites in Cluster 1B were hatcheries, predominantly medium to large size and mainly situated in densely populated poultry areas. They did not have susceptible birds on the site and had a high level of biosecurity, both in terms of confinement facilities and infrastructural hygiene standard. However, they generally had a relatively low score in relation to transportation hygiene standard and were characterized by relatively high frequencies of on-farm movements of animal products and off-farm movements of live birds.

3. Slightly reduced risk group - Cluster 3B $(n=203)$ : This cluster consisted of small hobby poultry premises that had susceptible birds, with very poor confinement facilities and poor infrastructural hygiene standard. However they had almost no on- and off-farm movements.

4. Slightly increased risk group - Cluster $3 \mathrm{~A}(n=78)$ : This cluster consisted predominantly of small hobby poultry premises (94\%) and small professional poultry farms with multiple production types/purposes (6\%). The majority of these sites had susceptible birds, with poor confinement facilities, a poor infrastructural hygiene standard and few on- and off-farm movements of live birds and fomites.

5. High risk group - Cluster 2A $(n=19)$ : This cluster was predominantly made up of small to medium size professional poultry farms (84\%) and hatcheries (16\%), most of them with keeping susceptible birds, having medium level standard confinement facilities and good infrastructural hygiene. These sites had high frequencies of on-farm movements of fomites and medium to high frequencies of off-farm movements of live birds and animal products.

6. Very high risk group - Cluster 2B $(n=16)$ : These sites were all professional poultry farms, mainly broiler farms (75\%). All kept susceptible birds, had medium-level standard confinement facilities, but scored lower with respect to infrastructure hygiene standard and exposure to visiting persons, in comparison with Cluster 2A. They also reported high frequencies of on- and off-farm movements of live birds and fomites.

\section{Discussion}

Designing a study from which conclusions could be drawn on the interactions between professional and hobby poultry populations, posed a unique challenge, since registers of hobby poultry premises in Belgium, as in most countries, do not exist. The results of this study made it possible to gain a first insight into the population density, characteristics and practices of hobby poultry sites. In the selected municipalities, around $9 \%$ of households keep hobby poultry at home. The density of hobby poul- try premises around professional operations was high, with the densest areas having between 8 and 22 premises per $\mathrm{km}^{2}$. However, these figures need to be interpreted with care since they are based on a non-random selection of 55 municipalities out of a total of 590 municipalities in Belgium. It is unlikely that these numbers are valid for the whole of Belgium since more rural than urban municipalities were present in the used dataset. It is believed that higher numbers of hobby poultry are present in these rural municipalities in comparison to the more urban regions.

These results clearly show that professional and hobby poultry farms are located in the vicinity of each other. A study of contacts between professional and hobby poultry farms in Switzerland found similar results, with high density areas with more than 8 poultry sites per $\mathrm{km}^{2}$ (Fiebig et al., 2009).

The stratified selection of the professional farms, according to bird species, production type and region, made it possible to include most types of farms in the sample. However, given that only 80 professional farms were contacted, some strata actually had too little farms in the sample to be representative. Therefore, the uncertainty of the results of these strata may be high. The response rates of $61.5 \%$ for professional poultry farms and $59.4 \%$ for hatcheries exceed other similar surveys (Hurnik et al., 1994; Fiebig et al., 2009). However, the response rate of $19.6 \%$ of hobby poultry keepers was rather low, which was partly due to the fact that a lot of participants encountered difficulties to connect to the internet site. The main advantage of a self-completed questionnaire online, as was done for the 2000 hobby poultry premises, is that it makes a survey in a large population very manageable. Possible disadvantages include that for non-responders no information could be collected about the reason for non-response. The data is therefore likely to be affected by a response-bias.

This study used two complementary approaches to deal with the problem of large numbers of independent variables (35 variables were used to evaluate biosecurity in this study) (Dohoo et al., 1997). First, variables linked to the same kind of risk were combined. In this, it was decided to assign 'hatching eggs' to the 'low susceptible species' group. Although HPAI virus may contaminate the surface of hatching eggs, it was assumed that the length of the hatching process and strict hygiene measures ensure that the virus does not survive the hatching process and therefore cannot act as a source of infection for one-day-old chicks (expert opinion)

The assumption that all potential biosecurity measures are equally weighted in the scoring system ( $1 / 0$ each variable) could be challenged, as some measures might play a more prominent role in reducing the risk of disease introduction and spread than other measures. However, for the vast majority of the different biosecurity measures suggested at present there is no data available on which a meaningful weighting could be based (Hagenaars, 2008). Therefore this study aimed at creating a linear scoring system whereby poultry sites can be compared relative to each other, rather than creating a quantitative scoring system defining biosecurity in absolute terms.

The level of biosecurity was, not surprisingly, associated with the type of poultry production (hobby versus 
professional). The extensive scale of hobby poultry facilities does not require the implementation of e.g. boot dips and sanitary transition zone. But their low confinement against the outdoor environment facilitates vector access to domestic poultry and thus increased the risk of disease dissemination. An interesting result is also the fact that the processing of kitchen waste is indicated as the most common reason for keeping birds by hobby poultry farmers. This is likely a result of several initiatives and incentives by local governments to use poultry to reduce the amount of waste produced by households. Yet this might harbour a certain risk since feeding of kitchen waste can also be a disease transmission route. It is sometimes stated that smaller conventional sites do not have a great motivation to implement preventive measures, because the costs would be relatively small if a new pathogen was introduced (Boklund et al., 2004). However, this study explored the attitude of hobby poultry keepers to mandatory measures (creating a covered and fenced outdoor scavenging area or to put all poultry indoors) and showed that a positive attitude towards preventive measures did exist. This attitude is probably influenced by the information campaign implemented by the media and the government, highlighting the risk of avian influenza introduction for animal and human health.

Larger facilities are often assumed to implement more advanced biosecurity measures, but the intensity of their operation also poses higher risks for infection and pathogen multiplication and dissemination. Although Belgian professional poultry farms and hatcheries have in general an acceptable level of adoption of standard biosecurity practices, further enhancement of their preventive measures is still possible. For example, a high proportion of the hatcheries (68\%) also had live poultry production activities at the hatchery site. In addition, $53 \%$ of them visited two or more farms per day with the same transport vehicle and $50 \%$ even used the same vehicle for multiple purposes. The small number of hatcheries purchasing hatching eggs from multiplier farms and selling one-day old chicks to broiler and rearing farms, may act as a bridge between otherwise separate sectors of the industry. When hatcheries also have live poultry production activities (susceptible species) on site, this source of pathogen transmission (through transportation) might become even more important. On top of this: $37 \%$ of the hatcheries had staff that had contact with other poultry farms or had poultry at home, which is something that should be avoided. Similarly, multi-species sites or multiple-production type sites are also at higher risk. One potentially underutilized practice in professional poultry farms appeared to be the provision of company clothing when supply or discharge teams and control agencies enter their facilities. Common service providers routinely contact different types of farms over wide areas. They therefore expose themselves to as many pathogens, including potentially AI viruses, which may survive for a moderate time at ambient temperatures on organic material, so that infection could be disseminated over large distances by the movement of service providers.

The intensity and frequency of different contacts between farms determines to a large extent the risk of spreading infectious agents. However, Belgium does not have an official database recording all on- and off- movements for poultry farms. This study gives a first overview of the intensity and frequency of poultry farm movements, by using information from a relatively small survey. Since data was received from 37 farms, the uncertainty for being able to make generalised inferences associated with the movement results for some farm types may be high. This study identified considerable variation in the movements and in the structure of the networks arising from these movements. Movement frequencies were higher at professional farms compared to hobby farms. Results showed that multiple production type farming systems had the highest total movement frequencies. Hatcheries also had high total movement frequencies. Yet, the average contact patterns that emerged do not necessarily reflect the reality of the underlying highly inter-dependent pattern of activities for an individual holding. Therefore more detailed study is required to further describe these patterns.

Monthly frequencies of professional visits often exceeded those of poultry and egg movements. Such contacts may be a commonly overlooked means of disease transmission among facilities and provide evidence that increased biosecurity awareness is essential.

Although hobby poultry keepers practiced less biosecurity measures in general, they moved birds very infrequently. Professional and hobby poultry sites were connected, but movements of poultry and eggs were found only to occur from professional to hobby farms and not in the other direction. Yet, hobby poultry keepers purchased poultry and eggs from the professional poultry site and in doing so might pose a risk of indirect disease transmission. Further connections were found through farms visiting the same bird shows. These results are comparable to the findings of the work by Fiebig et al. (2009) in Switzerland. Thus, the common assumption of a closed circuit of the professional poultry production without any connections to hobby farms does not entirely hold true (Bavinck et al., 2009).

An important result of this study is the identification and characterisation of the different clusters of farms existing in Belgium. The practices investigated can be assumed to have a potential influence on the introduction and spread of contagious diseases. The characterisation of poultry farms into different clusters also represents differences in disease spread and control. The result of this study may be useful for mathematical models of pathogen transmission between farms and aid the development of surveillance programs and tailored recommendations for farmers.

\section{Conclusions}

In conclusion, this study has shown that high densities of hobby poultry sites coexist with professional poultry farms and that links between the professional poultry sector and hobby poultry sites do exist. This might represent a risk for the spread of infectious agents between these two compartments. Therefore all types of poultry sites, irrespective of whether professional or not, should be provided with tailored recommendations and also need to be appropriately represented in models simulating poultry disease spread. Six groups of poultry sites were differentiated and 
risk-classified according to their risk of disease introduction and spread.

\section{Acknowledgments}

We thank the farmers for their willingness to be interviewed in the study. This study was funded by the Federal Public Service of Health, Food Chain Safety and Environment, Belgium (Contract RF 6192 AIRISK).

\section{References}

Alexander, D.J., 2007. An overview of the epidemiology of avian influenza Vaccine 25, 5637-5644.

Bavinck, V., Bouma, A., Van Boven, M., Bos, M.E.H., Stassen, E., Stegeman, J.A., 2009. The role of backyard poultry flocks in the epidemic of highly pathogenic avian influenza virus (H7N7) in the Netherlands in 2003. Prev. Vet. Med. 88, 247-254.

Boklund, A. Alban, L., Mortensen, S., Houe, H., 2004. Biosecurity in 116 Danish fattening swineherds: descriptive results and factor analysis. Prev. Vet. Med. 66, 49-62.

Calavas, D., Bugnard, F., Ducrot, C., Sulpice, P., 1998. Classification of the clinical types of udder disease affecting nursing ewes. Small Ruminant Res. 29, 21-31.

Costard, S., Porphyre, V., Messad, S., Rakotondrahanta, S., Vidon, H., Roger, F., Pfeiffer, D.U., 2009. Multivariate analysis of management and biosecurity practices in smallholder pig farms in Madagascar. Prev. Vet Med. 92, 199-209.

Dohoo, I.R., Ducrot, C., Fourichon, C., Donald, A., Hurnik, D., 1997. An overview of techniques for dealing with large numbers of independent variables in epidemiologic studies. Prev. Vet. Med. 29, 221-239.

East, I.J., 2007. Adoption of biosecurity practices in the Australian poultry industries. Aust. Vet. J. 85, 107-112.

Ferrari, P., Anonni, P., 2005. Missing data in optimal scaling. In: Lavoro Presentato al CLADAG 2005 , Universita di Milano, p. 9.

Fiebig, L., Smieszek, T., Saurina, J., Hattendorf, J., Zinsstag, J., 2009. Contacts between poultry farms, their spatial dimension and their relevance for avian influenza preparedness. Geospat. Health 4, 79-95.

Hagenaars, T.J., 2008. Between-farm Transmission Routes of Highly Transmissible Diseases in Livestock: A Literature Study on the Quantitative Knowns and Unknowns. Central Veterinary Institute of Wageningen, UR, Lelystad, p. 13.

Hurnik, D., Dohoo, I.R., Donald, A., Robinson, N.P., 1994. Factor analysis of swine farm management practices on Prince Edward Island. Prev. Vet. Med. 20, 135-146.

Köbrich, C., Rehman, T., Khan, M., 2003. Typification of farming systems for constructing representative farm models: two illustrations of the application of multi-variate analyses in Chile and Pakistan. Agric. Syst. 76, 141-157.

Kristensen, S.P., 2003. Multivariate analysis of landscape changes and farm characteristics in a study area in Central Jutland. Denmark. Ecol. Model. 168, 303-318.

Ligon, B.L., 2005. Avian influenza virus H5N1: a review of its history and information regarding its potential to cause the next pandemic. Semin. Pediatr. Infect. Dis. 16, 326-335.

Linting, M., Meulman, J.J., Groenen, P.J.F., 2007. Nonlinear principal components analysis: introduction and application. Psychol. Methods 12, 336-358.

Lyytikäinen, T., Kallio, E.R., 2008. Risk-classification of finnish pig farms by simulated FMD spread. In: Peelr, E.J., Alban, L., Russell, A., and the SVEPM Executive Committee (Eds.), Society for Veterinary Epidemiology and Preventive Medicine, Proceedings of a Meeting Held at Liverpook, UK, pp. 285-300.

Meuleman, J.J., van der Kooij, A.J., Heiser, W.J., 2004. Principal components analysis with nonlinear optimal scaling transformations for ordina and nominal data. In: Kaplan, D. (Ed.), Handbook of Quantitative Methods in the Social Sciences. Sage Publications, Newbury Park, CA, pp. 49-70.

Milán, M.J., Bartolomé, J., Quintanilla, R., Garcia-Cachán, M.D., Espejo, M., Herráiz, P.L., Sánchez-Recio, J.M., Piedrafita, J., 2006. Structural characterisation and typology of beef cattle farms of Spanish wooded rangelands (dehesas). Livest. Sci. 99, 197-209.

Nespeca, R., Vaillancourt, J.P., Morgan Morrow, W.E., 1997. Validation of a poultry biosecurity survey. Prev. Vet. Med. 31, 73-86.

Niemi, J.K., Lyytikäinen, T., sahlström, L., Virtanen, T., Lehtonen, H. 2009. Risk classification in animal disease prevention: who benefits from differentiated policy? In: Selected Paper Prepared for Presentation at the Agricultural and Applied Economics Association 2009 AAE and ACCI Join Annual Meeting, Milwaukee, Wisconsin, p. 28.

Ortiz-Pelaez, Á., Pfeiffer, D., 2008. Use of data mining techniques to investigate disease risk classification as a proxy for compromised biosecurity of cattle herds in Wales. BMC Vet. Res. 4, 24.

Ribbens, S., Dewulf, J., Koenen, F., Mintiens, K., De Sadeleer, L., de Kruif, A. Maes, D., 2008. A survey on biosecurity and management practices in Belgian pig herds. Prev. Vet. Med. 83, 228-241.

Rose, N., Madec, F., 2002. Occurrence of respiratory disease outbreaks in fattening pigs: relation with the features of a densely and a sparsely populated pig area in France. Vet. Res. 33, 179-190.

Sharkey, K.J., Bowers, R.G., Morgan, K.L., Robinson, S.E., Christley, R.M., 2008. Epidemiological consequences of an incursion of highly pathogenic H5N1 avian influenza into the British poultry flock. Proc. R. Soc. Lond. B Biol. Sci. 275, 19-28.

Solano, C., Bernués, A., Rojas, F., Joaquín, N., Fernandez, W., Herrero, M. 2000. Relationships between management intensity and structural and social variables in dairy and dual-purpose systems in Santa Cruz. Bolivia Agric. Syst. 65, 159-177. 